\title{
Distribuição geográfica potencial de espécies americanas do caranguejo "violinista" (Uca spp.) (Crustacea, Decapoda) com base em modelagem de nicho ecológico
}

\author{
João Carlos Nabout, Paulo de Marco Júnior, Luis Maurício Bini \& José Alexandre F. Diniz-Filho
}

Laboratório de Ecologia Teórica e Síntese, Departamento de Ecologia, ICB, Universidade Federal de Goiás, Caixa Postal 131, 74001-970

Goiânia, GO, Brasil. (naboutjc@ @otmail.com)

\begin{abstract}
Potential geographical distribution of American species of "fiddler crab" (Uca spp.) (Crustacea, Decapoda) based on ecological niche model. The genus $U c a$ Leach, 1814 (fiddler-crabs) is a well-know group characterized by a marked sexual dimorphism. Worldwide, there are 97 species of fiddler crabs described. Here, we predicted the potential geographical distribution of four species of this genus (Uca maracoani Latreille, 1802-1803, U. uruguayensis Nobili, 1901, U. panacea Novak \& Salmon, 1974 e U. monilifera Rathbun, 1914), which occur in the American continent coast. Data used in the modeling were compiled from occurrence records available in the literature. We used Maxent and GARP programs for modelling, 10 climatic variables and three topographical variables. All variables were converted to a grid resolution of 0.0417 degrees. In both Maxent and GARP models the predicted geographical distribution of the species was larger than suggested by other observed data works, except for U. monilifera. According to the AUC criterion, the models generated by GARP were better than those obtained from Maxent. Evaluating the two models together, it is recommended conservation plans for species with restricted habitat (U. panaceae and U. monilifera) besides suggesting sampling of $U$. maracoani in the Brazilian northeast and $U$. uruguyaensis in the Brazilian southeast.
\end{abstract}

KEYWORDS. Garp, Maxent, sampling plans, conservation plans.

RESUMO. Caranguejos do gênero Uca Leach, 1814 (caranguejo "violinista") são um grupo bem conhecido e caracterizado por um acentuado dimorfismo sexual e assimetria da quela do macho. Atualmente, estão descritas 97 espécies no mundo. Objetivou-se estimar a distribuição geográfica potencial de 4 espécies do gênero Uca que ocorrem na costa do continente Americano: Uca maracoani Latreille, 1802-1803, U. uruguayensis Nobili, 1901, U. panacea Novak \& Salmon, 1974 e U. monilifera Rathbun, 1914. Para modelar a distribuição dessas espécies nas Américas foram utilizados pontos de ocorrência compilados da literatura. Para a modelagem foram utilizados os programas Maxent e GARP a partir de 10 variáveis climáticas e três variáveis topográficas. Todas as variáveis foram convertidas para uma malha com resolução de 0,0417 graus. Nos dois modelos (Maxent e GARP) as espécies apresentaram distribuição geográfica maior do que sugerido por outros trabalhos de registro de ocorrência, com exceção de $U$. monilifera. Segundo o critério de área sob a curva (AUC), os modelos gerados pelo GARP apresentaram melhores resultados do que os modelos do Maxent. Entretanto, avaliando em conjunto os resultados dos dois modelos é possível melhor estabelecer planos de conservação para espécies com habitat restrito ( $U$. panaceae e $U$. monilifera), além de recomendar um aumento na amostragem de $U$. maracoani no nordeste brasileiro e $U$. uruguyaensis no sudeste brasileiro, a fim de detectar possíveis aumentos na sua distribuição geográfica com base nas predições dos modelos de nicho.

PALAVRAS-CHAVE. Garp, Maxent, delineamento amostral, planos de conservação.

Caranguejos do gênero Uca Leach, 1814 (conhecidos como chama-maré ou caranguejo violinista) são localmente abundantes, habitando zonas de entremarés de estuários de clima tropical, subtropical e temperado quente (MASUnARI, 2006). Esse é um grupo bem conhecido, caracterizado por um acentuado dimorfismo sexual, com machos apresentando uma grande quela (que representa um terço da massa corpórea total) (Crane, 1975; Rosenberg, 2001). Atualmente, 97 espécies do gênero estão descritas (CRANE, 1975; ROSEMBERG 2001). Outros trabalhos têm abordado diferentes aspectos da biologia deste grupo, tais como estudos de comportamento, seleção sexual (Von HAGEN, 1987; BACKWEll et al., 1999) e morfometria (ROSENBERG, 1997). Uma revisão extensiva da biologia desse grupo é apresentada por ROSENBERG (2001).

A modelagem preditiva de distribuição geográfica vêm se tornando uma ferramenta importante em estudos de ecologia e conservação incluindo o estudo da expansão em espécies invasoras (PETERSON et al., 2007; ZHu et al., 2007), o prognóstico dos efeitos das mudanças climáticas sobre a biodiversidade (MANN, 2000) e planos para conservação de espécies ameaçadas (Solano \& FERIA, 2007). A modelagem preditiva de distribuição está baseada na determinação de funções que descrevam o nicho ecológico de espécies com base em pontos de ocorrência conhecidos e dados ambientais (GuIsan \& Zimmermann, 2000). Existem diversas técnicas que permitem essa modelagem do nicho, tais como modelos lineares generalizados (GLM, "Generalized Linear Models", McCullagh \& Nelder, 1989), modelos aditivos generalizados (GAM, "Generalized Additive Models", Hastie \& Tibshirani, 1990), análise de componentes principais (PCA, "Principal Component Analysis", ROBERTSON et al., 2003), redes neurais artificiais (ANNs, "Artificial Neural Networks", MASTRORILlo et al., 1997), máxima entropia (Maxent, "Maximum Entropy", PhiLliPs et al., 2006), dentre outras (Elith et al., 2006).

Apesar dos vários trabalhos relacionados com caranguejos do gênero Uca (ver Rosenberg 2001), nenhum enfocou a distribuição geográfica utilizando técnicas de modelagem de nicho. É importante ressaltar 
que os habitats das espécies costeiras estão ameaçados em função do aquecimento global, da fragmentação de habitat e da urbanização das regiões de mangue. Neste contexto, os modelos de distribuição de espécies são ferramentas importantes para conservação das espécies (CARroll et al., 2001), permitindo uma melhor estimativa da distribuição geográfica das espécies e de padrões relacionados (e.g. riqueza, endemismo). Ademais, estudos com esse enfoque podem ser úteis para estabelecer locais para a criação de unidades de conservação e manejo.

O escopo deste trabalho foi estimar a distribuição geográfica potencial de quatro espécies de caranguejo do gênero Uca que ocorrem na costa do continente Americano. Especificamente, objetivou-se gerar mapas de distribuição potencial dessas espécies e indicar regiões de amostragem com base nos mapas gerados.

\section{MATERIAL E MÉTODOS}

As espécies analisadas neste estudo apresentam padrões distintos de distribuição geográfica no continente Americano: Uca maracoani Latreille, 1802-1803, U. uruguayensis Nobili, 1901 (ambas da costa Leste da América do Sul), U. panacea Novak \& Salmon, 1974 (costa Leste da América do Norte) e U. monilifera Rathbun, 1914 (costa Oeste da América do Norte) (Figs. 1-4). Os dados de ocorrência de todas as espécies, foram compilados a partir da literatura (CRANE, 1975; BRANCO, 1991; IRIBARNE \& MARTINEZ, 1999; RoBALDO \& MONSERRAT, 1999; ARMENDÁRIZI \& CÉSAR, 2002; JORDÁ \& RoCCATAGLIATA, 2002; RocCATAGLIATA \& JordÁ, 2002; ThURMAn, 2003; CÉsAR et al., 2005; MASUNARI et al., 2005; ALMEIDA et al., 2006; BEZERrA et al., 2006; MASUnARI, 2006). Este trabalho representa a compilação mais completa de informações desse grupo. A espécie $U$. maracoani foi registrada em 12 localidades, $U$. uruguayensis em 21 localidades, $U$. panacea em 10 localidades e U. monilifera em 2 localidades.

A seleção dessas espécies foi baseada na amplitude de sua distribuição geográfica de acordo com CRANE (1975), pois duas espécies possuem hábitat restrito ( $U$. panacea e $U$. monolifera) e duas com hábitat amplo ( $U$. maracoani e U. uruguayensis). Além disso selecionouse espécies de regiões costeiras do Leste e do Oeste do continente.

Para a modelagem foram utilizados dois programas que utilizam procedimentos de otimização a fim de relacionar a presença das espécies com características do ambiente: o Maxent ("Maximum Entropy") (PHILlips et al., 2006), que utiliza o método de máxima entropia, e o GARP ("Genetic Algorithm for Rule-set Production"), com base em algoritmos genéticos (STOCKWELl \& Peters, 1999).

O Maxent é um programa recente e tem se apresentado melhor que outros programas para um conjunto de dados pequenos (PEARson et al., 2007), como o caso da espécie $U$. monolifera (com duas ocorrências). A técnica do Maxent é um método de aplicação geral para fazer previsões ou inferências a partir de informações incompletas (Phillips et al., 2006). Este método parte do conceito que a distribuição que se conhece de uma espécie é a representação adequada do seu nicho ecológico, a partir dai o método atribui valores de zero e um para os demais quadrantes (pixels) da área geográfica, identificando quanto de erro estão em relação ao modelo ideal. Diferentemente de outros métodos, o Maxent modela a distribuição das espécies apenas com dados de presença. Os parâmetros utilizados para o modelo do Maxent foram os padrões (default), exceto por mil interações remoção de duplicatas.

Por outro lado, o GARP emprega vários métodos individuais (e.g., BIOCLIM, regressão logística) e utiliza as combinações das habilidades analíticas desses métodos para criar e otimizar um conjunto de regras. Esse algoritmo genético tenta encontrar relações não aleatórias entre os dados de ocorrência da espécie com as características ambientais do local do registro, e produz um modelo da distribuição potencial do organismo (STOCKWEll \& Noble, 1991). Na otimização, cada espécie foi processada 200 vezes, com 2.000 interações em cada rodada. O limite de convergência foi igual a 0,001. Foram selecionados os 20 melhores modelos gerados para cada espécie, sendo $0 \%$ para erro de omissão extrínseca e $10 \%$ para erro de comissão.

As seguintes variáveis ambientais foram utilizadas para a modelagem: precipitação no trimestre mais frio, precipitação no trimestre mais quente, coeficiente de variação da precipitação, precipitação anual, temperatura média do trimestre mais seco, temperatura média do trimestre mais úmido, temperatura máxima do período mais quente, temperatura mínima do período mais frio, coeficiente de variação da temperatura, temperatura média anual (Hijmans et al., 2005), altitude, inclinação e aspecto (USGS, HYDRO1k Elevation Derivative Database). Todas as variáveis foram convertidas para uma malha com resolução de 0,0417 graus.

Os modelos gerados pelo Maxent e pelo GARP foram avaliados selecionando um limiar mínimo ("mininum threshold") para determinar a presença potencial da espécie. Para esta avaliação dos modelos, uma amostra de pontos de ocorrência da espécie é classificada como presença ou ausência e comparada com os dados observados por uma matriz de confusão (MANEL et al., 2001). Dessa forma, a sensitividade do modelo é definida como a proporção de presenças verdadeiras em relação ao total de presenças preditas pelo modelo, enquanto que a especificidade do modelo é a proporção de ausências verdadeiras em relação ao total de ausências preditas pelo modelo. Assim, o ROC ("Receiver Operating Characteristics") é obtido relacionando-se sensitividade com o complemento da especificidade, resultando em uma probabilidade do limiar mínimo. Esse procedimento é um método para a avaliação independente do modelo (MANEL et al., 2001). A área abaixo da curva (AUC, "area under the curve") obtida a partir da integração da curva ROC foi usada para avaliar os modelos de distribuição de espécies. O AUC varia de zero a um, no qual valores próximos a um indicam alto desempenho do modelo, enquanto que valores menores do que 0,5 indicam baixo desempenho do modelo (Allouche et al., 2006; Elith et al., 2006). Como freqüentemente não se tem dados de real ausência das espécies, PHILliPs et al. (2006) gerou uma amostra de mil pontos de pseudo-ausências para juntar à amostra e estimar a curva ROC e AUC para o Maxent. Repetiu-se o mesmo procedimento para o GARP para permitir a comparação entre os métodos. Também registrou-se, para cada espécie a área da ocorrência predita 
pelo GARP e Maxent, usando o limiar mínimo para "cortar" a distribuição potencial. O segundo autor gerou um programa em SCILAB (CAMPBELL et al., 2006) para a obtenção do limiar mínimo e AUC para o GARPe Maxent.

\section{RESULTADOS E DISCUSSÃO}

As regiões de mangue sofrem grande impacto ambiental, principalmente devido ao avanço da urbanização, provocando assim a fragmentação de habitat. Dessa forma, torna-se importante o estudo da distribuição geográfica de espécies de mangue, sendo de fundamental importância a criação de planos para conservação desse tipo de habitat (MANN, 2000).

A espécie $U$. maracoani ocorre ao longo de todo litoral brasileiro até a Venezuela, mas tanto o modelo do Maxent (Fig. 5) quanto o do GARP (Fig. 9) indicaram que essa espécie apresenta uma área de distribuição potencialmente maior (CRANE, 1975; MASUNARI, 2006; Bezerra et al., 2006). O Maxent apresentou maior
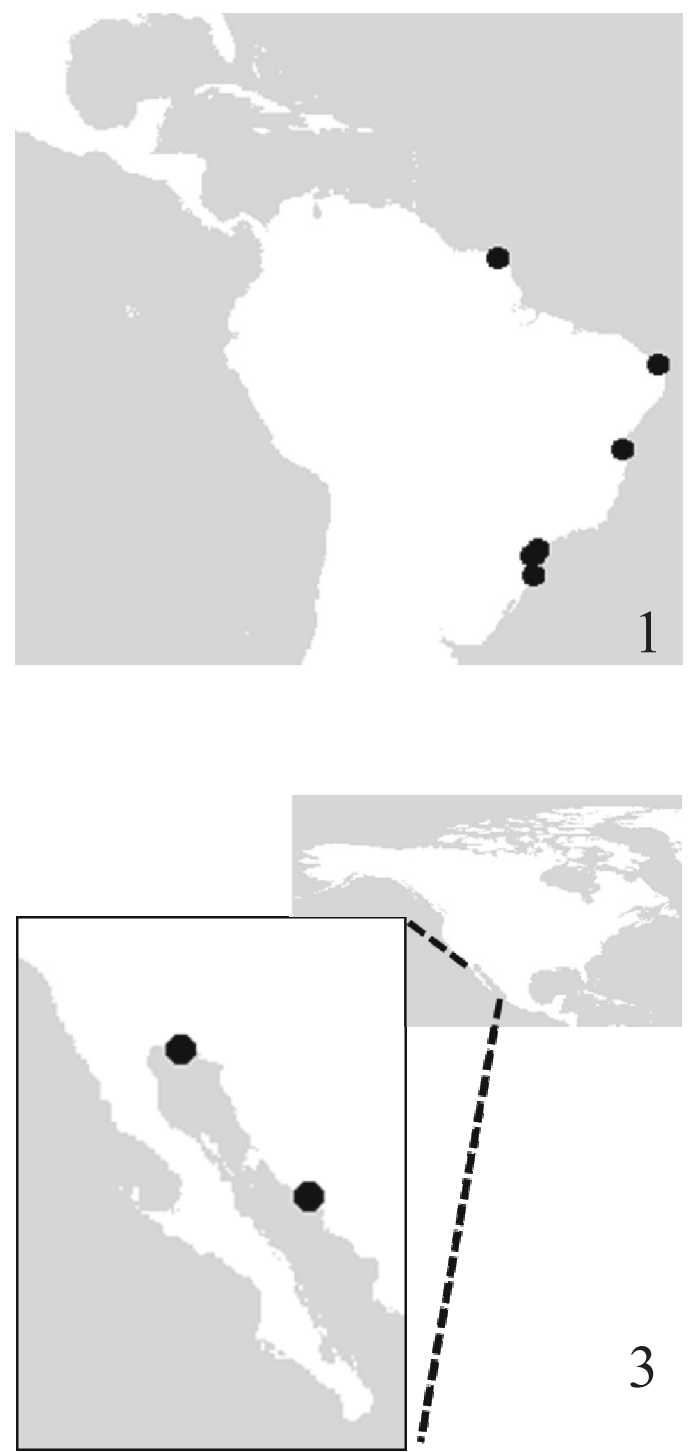

distribuição dessa espécie, indicando a ocorrência desta até mesmo em parte do Golfo do México, no Golfo de Honduras e no Golfo do Guayaqui (Oceano Pacífico). Os modelos do Maxent (Fig. 6) e do GARP (Fig. 10) geraram distribuição potencial similar para $U$. uruguayensis, e ambos os modelos foram semelhantes ao que foi predito por CRANE (1975) (distribuição concentrada na região Sul do Brasil até o Uruguai). Ámbas as espécies, apesar de terem distribuição ampla segundo os dois modelos, estão inseridas em regiões de grande impacto ambiental. Segundo MYers et al. (2000), a região do litoral brasileiro é considerada uma região de elevado endemismo e grande impacto antrópico (hotspot mundial).

De acordo com CRANe (1975), a espécie $U$. monilifera é geograficamente restrita, com registro de ocorrência somente na região do Golfo da California. O Maxent (Fig. 7) e o GARP (Fig. 11) corroboram essa distribuição indicando que essa espécie tem uma distribuição extremamente restrita, ocorrendo somente no extremo norte do Golfo da Califórnia. No entanto, segundo
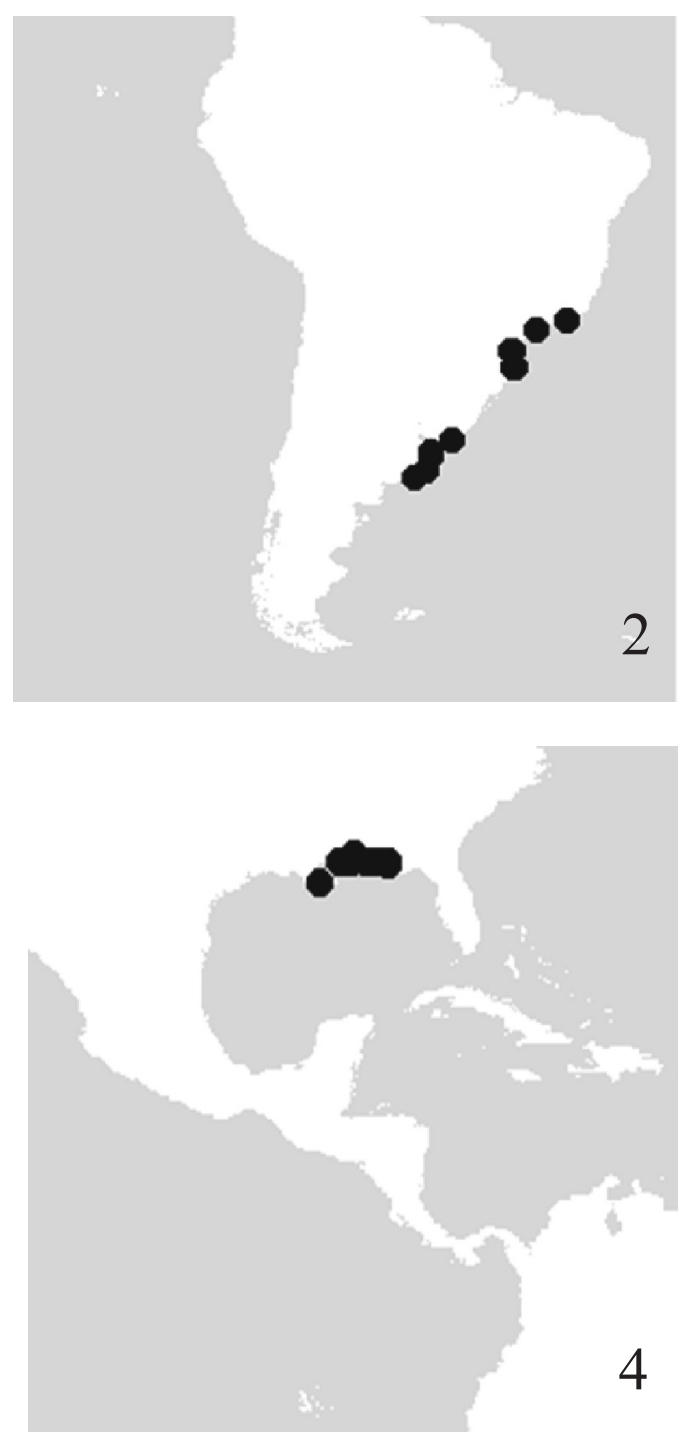

Figs. 1-4. Localização das espécies estudadas: 1, Uca maracoani Latreille, 1802-1803; 2, Uca uruguayensis Nobili, 1901; 3, Uca monilifera Rathbun, 1914; 4, Uca panacea Novak \& Salmon, 1974 (os círculos em negrito indicam os registros de ocorrência utilizados para a modelagem de nicho ecológico). 
o GARP, essa espécie tem distribuição muito mais restrita do que indicado pelo Maxent, que parece ter gerado um modelo melhor que o GARP. Por fim, os dois modelos apresentaram resultados semelhantes para $U$. panacea (Figs. 8, 12), ocorrendo principalmente no Golfo do México (Baia de Apalanches). Assim, isso sugere a importância de estabelecer ações prioritárias para conservação dessas espécies (principalmente $U$. monilifera, que apresentou distribuição extremamente restrita).

Alguns autores têm investigado as causas da distribuição espacial de caranguejos $U c a$, e sabe-se que inúmeras variáveis abióticas influenciam a distribuição destes animais, tais como: temperatura e salinidades (CRANe 1975), tipo de solo e substrato (Thurman, 1987; RiBEIRO et al., 2005), nível do substrato em relação à altura da maré (ThuRMAN, 1987) e presença de vegetação (NoBbS, 2003).

Segundo Bogazzi et al. (2001), a distribuição geográfica de $U$. uruguayensis é explicada pelo padrão dos ventos e pela influência das correntes oceânicas na distribuição de larvas dessa espécie. Embora haja importância das variáveis ambientais oceânicas na distribuição geográfica das espécies estudadas, os modelos gerados nesse trabalho utilizaram somente variáveis ambientais terrestres, e ainda assim a potencial distribuição geográfica de $U$. uruguayensis obtidas foram satisfatórias. BEZERRA et al. (2006) investigaram a influência do substrato na distribuição geográfica de $U$. maracoani (encontrada em regiões abertas e de substrato arenoso fino) e não apresentaram nenhuma correlação com quantidade de matéria orgânica ou umidade. Da mesma forma como ocorreu com $U$. uruguayensis, as variáveis terrestres foram suficientes para descrever bem a distribuição de $U$. maracoani.

Para as espécies de distribuição restrita as variáveis ambientais terrestres também foram suficientes para descrever bem a potencial distribuição dessas espécies. A espécie U. panacea, está limitada á região do Golfo do México (região semi-árida), sendo que essa espécie apresenta adaptações para sobreviver em regiões de altas temperaturas (e.g., a baixa perda corpórea de água) (Thurman, 1998). Por fim, não existe nenhum trabalho discutindo a distribuição geográfica de $U$. monilifera (restrita a região do Golfo da Califórnia), possivelmente relacionada às condições únicas daquele ambiente.

Recentemente, a eficiência dos modelos gerados pelo GARP e Maxent têm sido testada usando a estatística AUC (ver PETERSON et al., 2007). Os modelos derivados do algoritmo genético (GARP) apresentaram maiores valores de AUC para todas as espécies, sendo que o maior valor foi igual a um para $U$. monilifera. O maior valor de AUC para o Maxent ocorreu também com a espécie $U$. monilifera $(0,9996)$, enquanto que o menor valor foi de 0,7671 para U. uruguayensis. Ainda assim, todos os valores de AUC obtidos foram maiores do que esperado ao acaso $(0,5)$ (Tab. I).
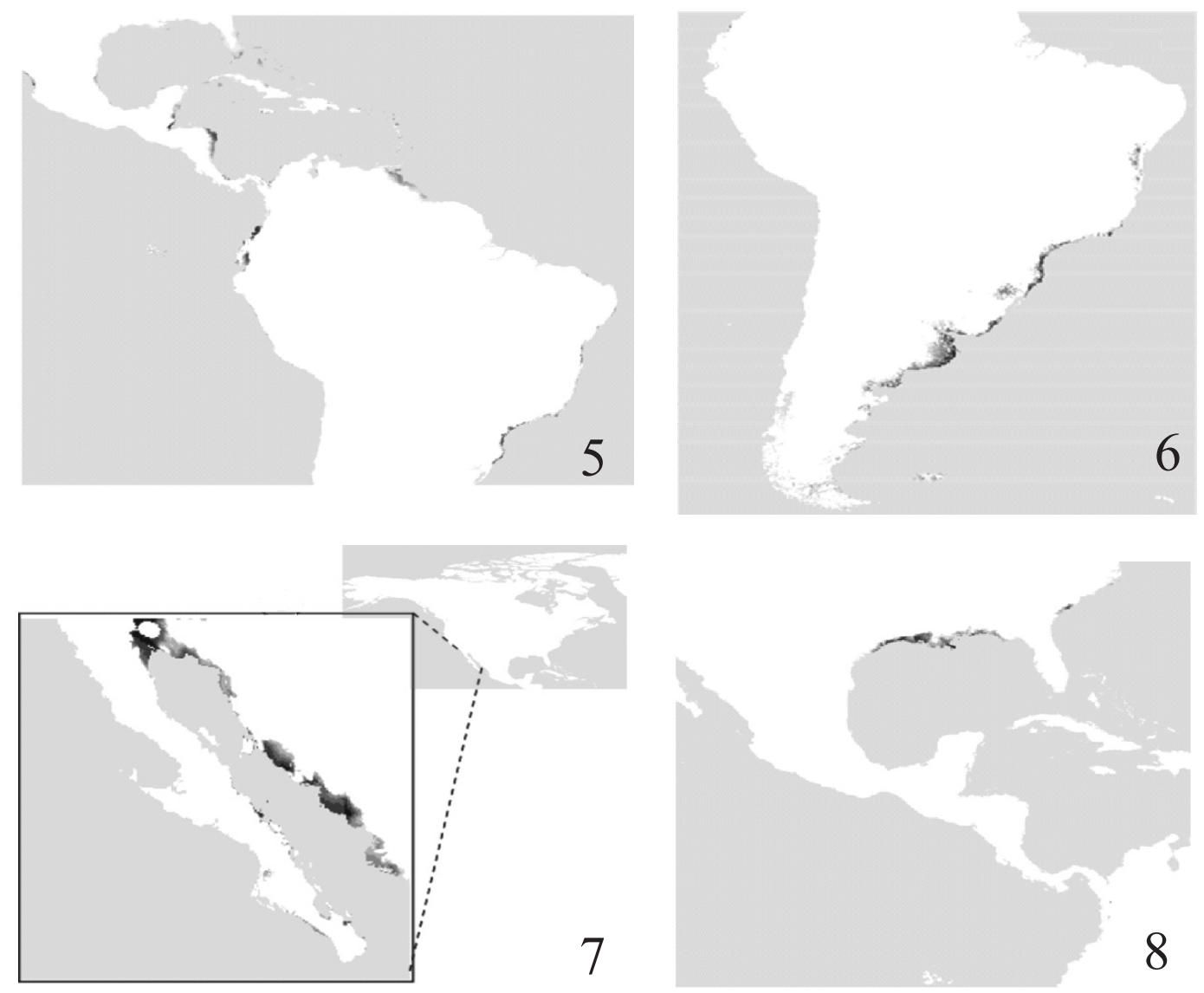

Figs. 5-8. Mapa de distribuição geográfica potencial predito pelo Maxent: 5, Uca maracoani Latreille, 1802-1803; 6, Uca uruguayensis Nobili, 1901; 7, Uca monilifera Rathbun, 1914; 8, Uca panacea Novak \& Salmon, 1974 (gradiente cinza da região costeira indica a probabilidade de ocorrência da espécie, no qual, as áreas mais escuras indicam maior probabilidade de ocorrência). 
Dessa forma, para as espécies de $U c a$ avaliadas neste trabalho, o GARP apresentou melhores resultados do que o Maxent, de acordo com os valores de AUC e ROC. A despeito da elevada freqüência de utilização do GARP, trabalhos recentes têm demonstrado que o Maxent apresenta melhores resultados (PhILliPs et al., 2004; ELITH et al., 2006; Phillips et al., 2006). Entretanto, para as espécies avaliadas neste estudo, o Maxent demonstrou ter superestimado a distribuição geográfica das espécies. ARAúJO \& NEW (2007) sugerem a utilização simultânea dos modelos, assim as previsões geradas pelo Maxent e GARP para as quatro espécies de caranguejo Uca podem ser usadas em conjunto para melhor descrever a distribuição geográfica potencial.

Todas as espécies avaliadas neste estudo tiveram distribuições geográficas potenciais semelhantes daquelas apresentadas no trabalho de CRANE (1975)

Tabela I. Avaliação dos modelos do Maxent e Garp (estimativa da distribuição geográfica de quatro espécies do gênero $U c a$ ) baseado no valor de AUC ("area under the curve") e ROC ("Receiver Operating Characteristics").

\begin{tabular}{lccrc}
\hline & $\begin{array}{c}\text { AUC } \\
\text { Maxent }\end{array}$ & $\begin{array}{c}\text { AUC } \\
\text { Garp }\end{array}$ & $\begin{array}{c}\text { ROC } \\
\text { Maxent }\end{array}$ & $\begin{array}{c}\text { ROC } \\
\text { Garp }\end{array}$ \\
\hline U. monilifera & 0.9996 & 1.0000 & 66.2187 & 0.0000 \\
U. panacea & 0.9112 & 0.9993 & 39.8085 & 0.0000 \\
U. maracoani & 0.9192 & 0.9991 & 0.0000 & 9.0000 \\
U. uruguayensis & 0.7838 & 0.9903 & 35.7542 & 0.0000 \\
\hline
\end{tabular}

baseado apenas nos registros de ocorrência. Diferentes fatores podem ter gerado as diferenças entre as distribuições observadas (ou pelo menos sua extensão, com base nos registros) e a distribuição potencial. De modo geral, elas podem refletir falhas no conhecimento (de modo que a falha estaria nos dados observados) ou podem refletir sobre-estimativas na ocupação do nicho da espécie. Esta, por sua vez, pode ser conseqüência do fato de que não é possível incorporar nos modelos todos os fatores ecológicos e históricos que poderiam explicar a distribuição geográfica de uma espécie.

Dessa forma, o modelo gerado reflete o nicho potencial das espécies e pode ser um indicativo de limitações nos processos de dispersão ou das interações entre espécies. Ou seja, modelos que predizem áreas maiores do que aquela onde a espécie de fato ocorre (e.g. para $U$. maracoani) podem indicar limitação na dispersão das espécies. Ainda, os modelos gerados não consideram as interações entre as espécies, ou seja, são nichos grinelianos (ver SOBERón, 2007). Por conseguinte, nichos grinelianos devem produzir modelos mais amplos.

Finalmente, é importante notar que frente às conseqüências do aquecimento global, o aumento do nível do mar tem sido caracterizado como uma forte ameaça aos ecossistemas costeiros, sendo que, nos últimos 15 anos, a taxa de aumento do nível do mar foi de $3,1 \mathrm{~mm}$ por ano (IPCC, 2007). O aquecimento global pode também acarretar mudanças na circulação dos oceanos e,
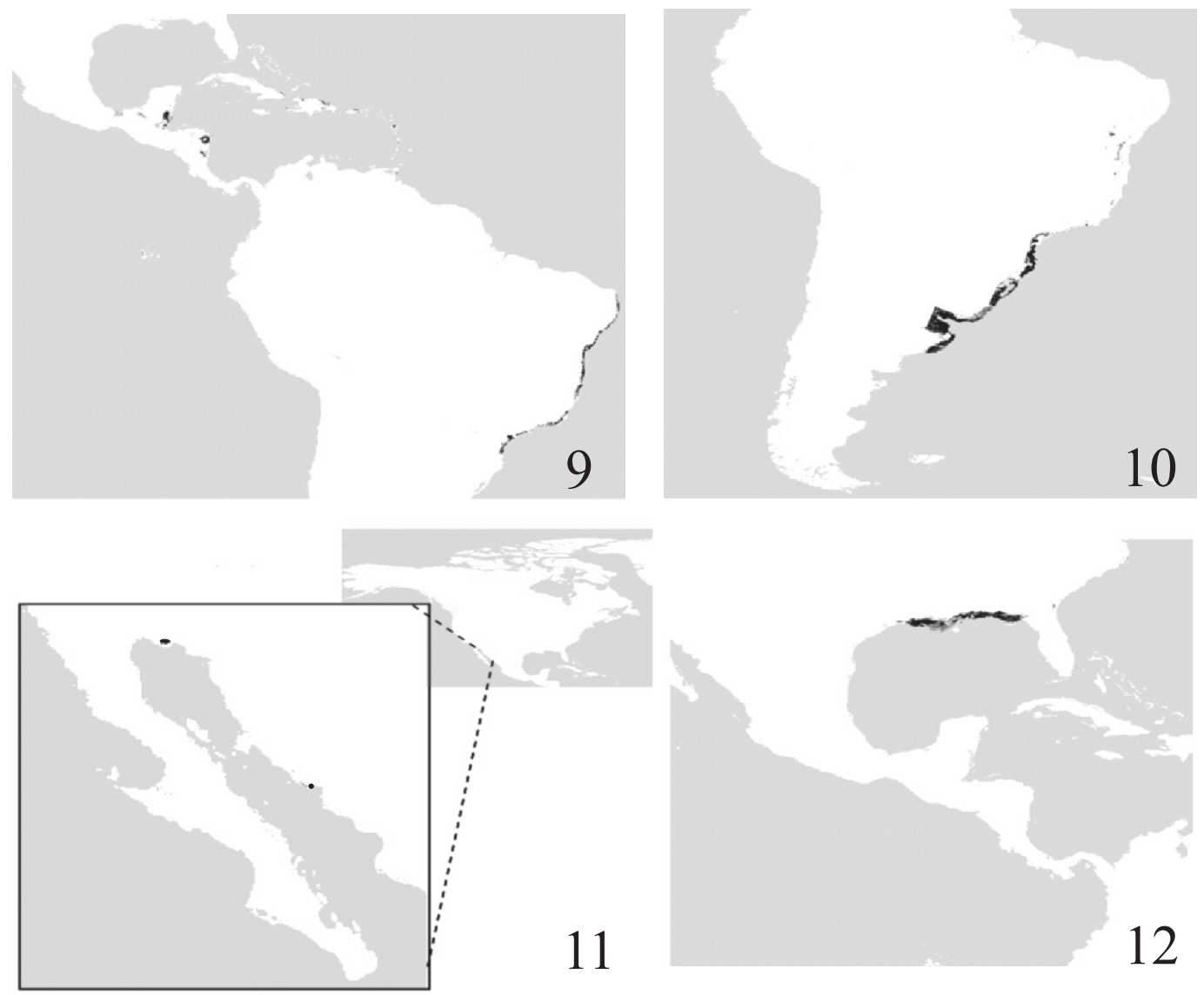

Figs. 9-12. Mapa de distribuição geográfica potencial predito pelo GARP: 9, Uca maracoani Latreille, 1802-1803; 10, Uca uruguayensis Nobili, 1901; 11, Uca monilifera Rathbun, 1914; 12, Uca panacea Novak \& Salmon, 1974 (gradiente cinza da região costeira indica o número de modelos que sugerem a ocorrência da espécie, no qual, as áreas mais escuras indicam maior número de modelos). 
consequente, alterar a ecologia dos organismos marinhos e costeiros (Gray, 1997; MAnN, 2000). Portanto, recomendam-se planos especiais de conservação para espécies com habitat restrito, como $U$. monilifera e $U$. panacea, pois essas podem sofrer maiores impactos provocados pelo aquecimento global e conseqüente elevação do nível do mar.

Os modelos apresentados aqui podem ser úteis para auxiliar na elaboração desses planos, utilizando modelos construídos com base em dados atuais a fim de projetar a distribuição potencial das espécies sob diferentes cenários de mudança climática. Entretanto, especialmente para espécies de distribuição costeira, ainda é preciso entender melhor os fatores limitantes associados à distribuição geográfica das espécies. Em acréscimo, este estudo demonstrou que a modelagem ecológica do nicho pode ser uma ferramenta computacional importante para complementar os estudos de delineamento amostral de espécies litorâneas e pode ser usado para identificar áreas prioritárias para conservação de espécies ameaçadas.

Agradecimentos. Esse trabalho foi realizado com o apoio da CAPES e CNPq por meio de concessão de bolsas de doutorado (JCN) e produtividade (PDMJ, LMB e JAFDF) respectivamente. Agradecemos ao prof. Dr. Laerte Ferreira Guimarães (LAPIG/ IESA/UFG), pelo incentivo e oportunidade de desenvolvimento deste trabalho e, a Ms. Levi Carina Terribile pelo auxílio com o GARP. Agradecemos ainda aos dois revisores (entre eles Adriano Sanches Melo da UFRGS) pelas valiosas contribuições.

\section{REFERÊNCIAS BIBLIOGRÁFICAS}

Allouche, O.; Tsoar, A. \& Kadmon, R. 2006. Assessing the accuracy of species distribution models: prevalence, kappa and the true skill statistic (TSS). Journal of Applied Ecology 43: $1223-1232$

Almeida, A. O.; Coelno, P. A.; Santos, J. T. A. \& Ferraz, N. R. 2006. Crustáceos decápodos estuarinos de Ilhéus, Bahia, Brasil. Biota Neotropica 6(2):1-24.

AraúJo, M. B. \& New, M. 2007. Ensemble forecasting of species distributions. Trends in Ecology and Evolution 22:42-47.

ArmendÁRIZ, L. C. \& CÉSAR, I. I. 2002. Estimación de la producción secundaria de Uca uruguayensis (Decapoda, Brachyura, Ocypodidae) en Bahía Samborombón, Argentina. Ciencias Marinas 32(3):551-558.

Backwell, P. R.; Jennions, Y. M. D.; Christy, J. H. \& Passmore, N. I. 1999. Female choice $n$ the synchronously waving fiddler crab Uca annulipes. Ethology 105:415-421.

Bezerra, L. E. A.; Dias, C. B.; Santana, G. X. \& Matthews-Cascon, H. 2006. Spatial distribution of fiddler crabs (genus Uca) in a tropical mangrove of northeast Brazil. Scientia Marina 70(4):759-766

Bogazzi, E.; Iribarne, O. O.; Guerrero, R. \& Spivak, E. 2001. Wind Pattern may explain the southern limit of distribution of a southwestern Atlantic fiddler crab. Journal Shellfish Research 20:353-360.

Branco, J. O. 1991. Aspectos ecológicos dos Brachyura (Crustacea, Decapoda) no manguezal do Itacorubi, SC - Brasil. Revista Brasileira de Zoologia 7(1-2):165-179.

Campbell, S.; Chancelier J. P. \& Nikoukhah R. 2006. Modeling and Simulation in Scilab/Scicos. New York, Springer. 313p.

Carroll, C.; Noss, R. F. \& Paquet, P. C. 2001. Carnivores as focal species for conservation planning in the Rocky Mountain region. Ecological Applications 11:961-980.

César, I. I.; Armendariz, L. C. \& Becerra, R. V. 2005. Bioecology of the fiddler crab Uca uruguayensis and the burrowing crab Chasmagnathus granulatus (Decapoda, Brachyura) in the Refugio de Vida Silvestre Bahía Samborombón, Argentina. Hydrobiologia, 545(1):237-248.

Crane, J. 1975. Fiddler crabs of the world, Ocypodidae: genus Uca. Princeton, Princeton University. 736p.
Elith, J.; Graham, C. H.; Anderson, R. P.; Dudik, M.; Ferrier, S.; Guisan, A.; Humans, R. J.; Huettmann, F.; Leathwick, J. R.; Lehmann, A.; LI J.; Lohmann, L. G.; Loiselle, B. A.; Manion, G.; Moritz, C.; NAKAMURA, M.; Nakazawa, Y.; Overton, J. M.; Peterson, T. A.; Phillips, S. J.; Richardson, K.; Scachetti-Pereira, R.; Schapire, R. E.; Soberon, J.; Williams, S.; Wisz, M. S. \& Zimmermann, N. E. 2006. Novel methods improve prediction of species' distributions from occurrence data. Ecography 29:129-151.

Gray, J. S. 1997. Marine biodiversity: patterns, threats and conservation needs. Biodiversity and Conservation 6(1): $153-175$.

Guisan, A. \& Zimmermann, N. E. 2000. Predictive habitat distribution models in ecology. Ecological Modeling 135:147-186.

Hastie, T. \& Tibshirani, R. 1990. Generalised Additive Models. London, Chapman and Hall. 355p.

Hijmans, R. J.; Cameron, S. E.; Parra, J. L.; Jones, P. G. \& Jarvis, A 2005. Very high resolution interpolated climate surfaces for global land areas. International Journal of Climatology 25: 1965-1978. Disponível em: <http://www.worldclim.org>. Acesso em: 18.12.2007.

USGS (U. S. Geological Survey). HYDRO1k Elevation Derivative Database. Disponível em: <http:// edcdaac.usgs.gov/gtopo30/hydro/>. Acesso em: 18.12.2007.

IPCC (Intergovernmental Panel on Climate Change). 2007. Climate Change 2007: The Physical Science Basis. Contribution of Working Group I to the Fourth Assessment Report of the Intergovernmental Panel on Climate Change. New York, Cambridge University. 996p.

Iribarne, O. O. \& Martinez, M. M. 1999. Predation on the Southwestern Atlantic Fiddler Crab (Uca uruguayensis) by Migratory $P$. squa tarola, Arenaria in terpres, Shorebirds (Pluvialis dominica, and Numenius phaeopus). Estuaries 22(1):47-54.

Jordá, M. T. \& Roccatagliata, D. 2002. Population dynamics of leidya distorta (Isopoda: Bopyridae) infesting the fiddler crab Uca uruguayensis at the rio de la plata estuary, argentina. Journal of Crustacean Biology 22(4):719-727.

Manel, S.; Williams, H. C. \& Ormerod, S. J. 2001. Evaluating presence-absence models in ecology: the need to account for prevalence. Journal Applied Ecology 38:921-931.

Mann, K. H. 2000. Ecology of Coastal Waters: with implications for management. Malden, Blackwell Science. 406p.

Mastrorillo, S.; Lek, S.; Dauba, F. \& Belaud, A. 1997. The use of artificial neural networks to predict the presence of smallbodied fish in a river. Freshwater Biology 38:237-246.

MasunARI, S. 2006. Distribuição e abundância dos caranguejos Uca Leach (Crustacea, Decapoda, Ocypodidae) na Baía de Guaratuba, Paraná, Brasil. Revista Brasileira de Zoologia 23(4):901-914.

Masunari, S.; Dissenha, N. \& FAlcão, R. C. 2005. Crescimento relativo e destreza dos quelípodos de Uca maracoani (Latreille) (Crustacea, Decapoda, Ocypodidae) no Baixio Mirim, Baía de Guaratuba, Paraná, Brasil. Revista Brasileira de Zoologia 22(4):974-983.

McCullagh, P. \& Nelder, J. A. 1989. Generalized Linear Models. 2nd ed. London, Chapman and Hall. 511p.

Myers, N.; Mittermeier, R. A.; Mittermeier, C. G.; Fonseca, G. A. B. \& Kent, J. 2000. Biodiversity hotspots for conservation priorities. Nature 403:853-858

NobBs, M. 2003 Effects of vegetation differ among three species of fiddler crabs ( $U c a$ spp.). Journal of Experimental Marine Biology and Ecology 284(29):41-50.

Pearson, R. G.; Raxworthy, C. J.; Nakamura, M. \& Peterson, A. T. 2007. Predicting species distributions from small numbers of occurrence records: a test case using cryptic geckos in Madagascar. Journal of Biogeography 34:102-117.

Peterson, A.; Williams, R. \& Chen, G. 2007. Modeled global invasive potential of Asian gypsy moths, Lymantria disparI. Entomologia Experimentalis et Applicata 125:39-44.

Phillips, S. J.; Anderson, R. P. \& Schapire, R. E. 2006 Maximum entropy modeling of species geographic distributions. Ecological Modeling 190:231-259.

Phillips, S. J.; Dudik, M. \& Schapire, R. E. 2004. A maximum entropy approach to species distribution modeling. In: BRODLEY, 
C. A. ed. International Conference on Machine Learning, $25^{\circ}$, Banff, 2004. Proceedings... Banff, Canada. 655-662p.

Ribeiro, P. D.; Iribarne, O. O. \& Daleo, P. 2005. The relative importance of substratum characteristics and recruitment in determining the spatial distribution of the fiddler crab Uca uruguayensis Nobili. Journal of Experimental Marine Biology and Ecology 14(1):99-111.

Robaldo, R. B. \& MonserRat, J. M. 1999. Larval occurence of Skrjabinoclava sp. (Nematoda: Acuarioidea) in the south american crab Chasmagnathus granulata and Uca uruguayensis. Atlântica, Rio Grande 21:5-10.

Robertson, M. P.; Peter, C. I.; Villet, M. H. \& Ripley, B. S. 2003 Comparing models for predicting species' potential distributions: a case study using correlative and mechanistic predictive modeling techniques. Ecological Modelling 164:153-167.

Roccatagliata, D. \& Jordá M. T. 2002. Infestation of the fiddler crab Uca uruguayensis by Leidya distorta (Isopoda, Bopyridae) from the rio de la Plata estuary, Argentina. Journal of Crustacean Biology 22(1):69-82.

Rosenberg, M. S. 1997. Evolution of shape differences between the major and minor chelipeds of Uca pugnax (Decapoda: Ocypodidae). Journal of Crustacean Biology 17:52-59. 2001. The systematics and taxonomy of fiddler crabs: a phylogeny of the genus Uca. Journal of Crustacean Biology 21(3):839-869.

Stockwell, D. R. B. \& Noble, I. R. 1991. Induction of sets of rules from animal distribution data: a robust and informative method of data analysis. Mathematics and Computers in
Simulation 32:249-254.

Stockwell, D. R. B. \& Peters, D. 1999. The GARP modelling system: problems and solutions to automated spatial prediction. International Journal of Geographical Information Science 13:143-158.

Soberón, J. 2007. Grinnellian and Eltonian niches and geographic distributions of species. Ecology Letters 10:1115-1123.

Solano, E. \& Feria, T. P. 2007. Ecological niche modeling and geographic distribution of the genus Polianthes L. (Agavaceae) in Mexico: using niche modeling to improve assessments of risk status. Biodiversity and Conservation 16:1885-1900.

Thurman, C. L. 1987. Fiddler crabs (Genus Uca) of eastern Mexico (Decapoda, Brachyura, Ocypodidae). Crustaceana 53(1):94-105. 1998. Evaporative Water Loss, Corporal Temperature and the Distribution of Sympatric Fiddler Crabs (Uca) from South Texas, Comparative Biochemistry and Physiology - Part A: Molecular \& Integrative Physiology 119(1):279-286.

2003. Osmoregulation by six species of fiddler crabs (Uca) from the Mississippi delta area in the northern Gulf of Mexico. Journal of Experimental Marine Biology and Ecology 291:233-253.

Von Hagen, H. O. 1987. Morphology and waving display of a new species of Uca (Crustacea, Brachyura) from the State of Espirito Santo (Brazil). Mitteilungen aus dem Hamburgischen Zoologischen Museum und Institut, Hamburg 84:81-94.

Zhu, L.; Sun, O. J.; SANG, E.; LI, Z. \& MA, K. 2007 Predicting the spatial distribution of an invasive plant species (Eupatorium adenophorum) in China. Landscape Ecology 22:1143-1154

Recebido em janeiro de 2008. Aceito em julho de 2008. ISSN 0073-4721

Artigo disponível em: www.scielo.br/isz 